\title{
La Isabela, un nuevo Real Sitio para los monarcas del siglo XIX
}

\author{
Amparo Aguado Pintor ${ }^{1}$
}

\begin{abstract}
RESUMEN ABSTRACT
El Real Sitio de La Isabela, afamado por sus baños termales, donde Fernando VII fundó una población de nueva planta, representa un hecho singular y anacrónico. Constituye un ejemplo tardío de urbanismo ilustrado en un Real Sitio, de envergadura semejante a los proyectos emprendidos en tiempos de Carlos III,

pero en una realidad politica bien distinta.

La Isabela fue concebida como nueva población en un Real Sitio que debia mantenerse habitado para funcionar como entidad benéfica, como lugar de ocio y reposo de los monarcas, y como núcleo de actividad económica, aunando el urbanismo cortesano de los Reales Sitios con otras experiencias de creación de poblaciones de nueva planta. Surgió en un periodo crucial durante el cual nuestro legado arquitectónico se

The Royal Country House of La Isabela, well-known for its thermal spa, where Fernando VII founded a village of new creation, represents an exceptional and anachronic fact. It constitutes a late sample of enlightened urbanism for a Royal Country Estate. This project has a similar magnitude to the ones undertaken by Carlos III, though in a very different political reality. La Isabela was concived as a new village within a Royal Country Estate, which should be inhabited to function as a charitable entity, a a pleasure and relax retirement place for the monarchy and as an economic nucleus. The place was designed to combine the courtly urbanism of the Royal Country Estates with other experiences concerned with the creation of new-planned villages. La Isabela was erected in the crucial
\end{abstract}

1 Aguado Pintor, Amparo: El Patrimonio Inmueble de la Corona en la Monarquia del siglo xix. El Real Sitio de La Isabela, un caso representativo. Tesis Doctoral. UNED, 25 de junio de 2001. 
vio radicalmente alterado en relación con la evolución de la Monarquia a lo largo del siglo xix y con el proceso desamortizador. Podemos observar, analizando su trayectoria, hasta qué punto incidieron en su decadencia las actitudes de una sociedad que se transformaba, la ineficacia de la normativa legal, el deterioro de los materiales, las mutaciones en la organización espacial y su viabilidad económica.

Refleja La Isabela en qué se transformó la refinada villa cortesana, creada en tiempos de los primeros Borbones, al pasar por el tamiz del cambio sociopolítico gestado a colnienzos del siglo $x I x$. period in which our architectural legate was radically altered by de monarchy's evolution through the XIX century and by the process of selling land from the Crown's heritage. Analizing the estate's course of development, we can observe how much its decay reponds to the actitudes of a society which was under the process of changing, the inefficiency of legal norm, the materials deterioration, the mutations in the spatial organization and its economic viavility.

La Isabela reflects wich was the outcome of a refined courtly village, created in the first Borbones, time when it through the sociopolitical changes taken place at the beginning of the xix century.

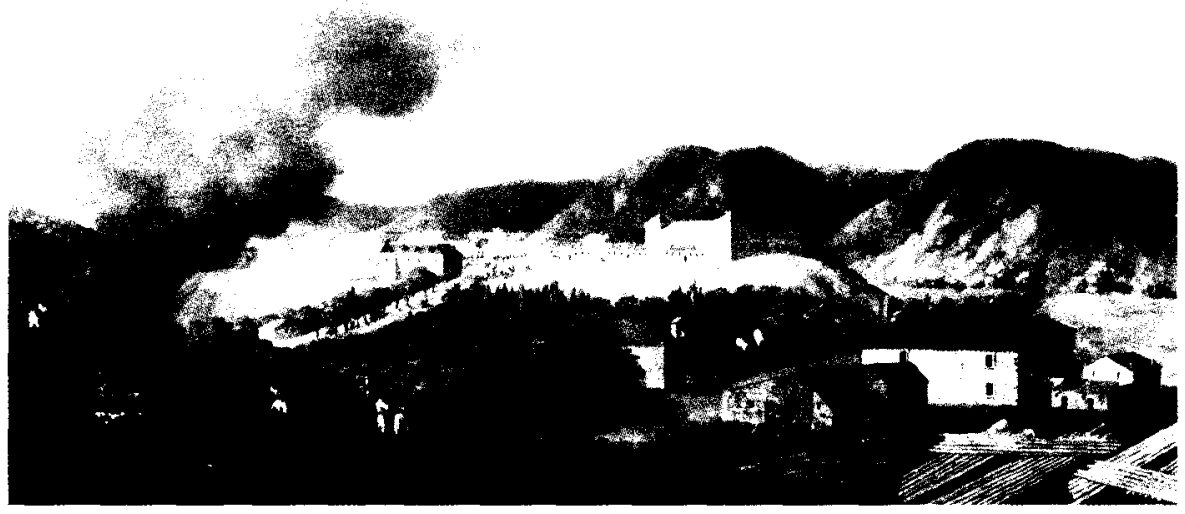

Fig. 1. Fernando Brambilla: Vista de La Isabela con el palacio y baños. CopyrightoPatrimonio Nacional.

El pintoresquismo de su emplazamiento, unido al hecho de que se tratase de un lugar con tradición histórica, características comunes a otros Reales Sitios, debieron contribuir en pro de la consideración especial que 
mereció La Isabela para Fernando VII e Isabel II. Pero también debieron influir poderosamente en ello sus posibilidades para ofrecer una nueva alternativa de ocio a los monarcas.

El sitio donde fue fundada $\mathrm{La}$ Isabela forma una pequeña península rodeada por el Guadiela al sureste de los confines de la provincia de Guadalajara y los septentrionales de Cuenca. En sus inmediaciones se distribuyen cerros y colinas de diversa elevación y se divisa en gran extensión la Sierra de Altomira.

Allí, a ocho kilómetros de la villa de Sacedón, se encontraban unos baños que fueron aprovechados desde la antigüedad. Hoy no es posible determinar la categoría que alcanzaron, pese a saber de la fama de que gozaron. Reafirmando lo que relatan las crónicas ${ }^{2}$, la remota utilización estos baños se ha confirmado, además de por las cualidades geológicas del entorno, por los vestigios hallados de población romana ${ }^{3}$.

Aunque el tiempo consumió la obra de los romanos, el eco de la fama de los baños de Sacedón alcanzó la época musulmana. Fueron reedificados el año $971{ }^{4}$ de modo que los baños se disponían en el contorno del estanque, que servía de depósito de las aguas, y se añadió la construcción de una casa, que proporcionaba alojamiento a los enfermos. Pero las construcciones para baños árabes solían ser arquitecturas frágiles de materiales perecederos, razón por la cual no se han conservado más vestigios que aquellos que permanecieron en la memoria colectiva.

En oposición a lo extendido de la misma en la cultura árabe, en el mundo cristiano la práctica balnearia se mantuvo de manera más discreta y evolucionó el uso de las instalaciones, que estarían configuradas por la construcción de un hospital, una casa de baños con una mínima

2 Fernando INFANTE: Teatro de la salud, baños de Sacedón hallados del D D. Fernando infante, médico de la Reina nuestra Señora, y añadido del doctor $D$. Juan de la Torre y Valcárcel (1663).

3 Realizando los trabajos de un caz se descubrieron restos de fábrica romana, habiéndose aprovechado para las obras del Real Patrimonio. Basilio Sebastián CASTELLANOS DE LOSADA (Madrid 1807-1891). Director de la Academia Española de Arqueología y Geografía, y del Museo Arqueológico Nacional, en su Manual del Bañista (Madrid, 1846) afirma también haber colocado en el museo de la Biblioteca Nacional algunos pavimentos de mosaico de una población situada al otro lado del río. En sus Apuntes para un catalogo de los objetos del Museo de la Biblioteca Nacional de Madrid, publicado en 1847, clasifica los iestos de pavimento con los números 281 y 282.

4 Marino PIzZI y Fragneschi: Tratado de las aguas medicinales de Salam-bir que comunmente llaman de Sacedón escrito en lengua árabe por Agmer Ben Abdala médico de Toledo en el año de mil cincuenta y cuatro; traducido al idioma castellano e ilustrado con varias notas... por ... Ma rino Pizzi y Fragneschi... Madrid, Antonio Pérez de Soto, 1761. 
infraestructura o por un monasterio ${ }^{5}$. Es frecuente la relación de fuentes y baños termales con monasterios del Císter por su vinculación atávica a los lugares de agua abundante. En el monasterio de Bernardos cercano a los Baños de Sacedón, donde habia otros pequeños manantiales, se veneraba la imagen de Nuestra Señora de Monsalud a quien se atribuían milagrosas curaciones. Es posible que, en ese contexto, durante el medievo, el foco de atracción que constituía el poder curativo de las aguas se trasladase de los antiguos baños al monasterio.

Y es que las aguas termales de los Baños de Sacedón, junto a los cuales fue construida La Isabela al comenzar el siglo XIX, se hallaban enclavadas en un entorno con condiciones naturales muy proclives a la aparición del fenómeno termal: Sacedón, Córcoles, Trillo y Buendía poseían manantiales con propiedades curativas ${ }^{6}$. Los Baños de Sacedón poseían la cualidad de estar convenientemente situados en un lugar de temperaturas no extremadas, buscando en verano la sombra y el frescor de las aguas, en una extensa planicie cercada por elevados cerros poblados por árboles y hierbas aromáticas, que le otorgaban una atmósfera sumamente sana.

La localización fue un factor determinante para esta nueva población por su doble carácter de balneario y de Real Sitio. Todos los Reales Sitios, independientemente de su función -explotación agropecuaria, aprovechamiento forestal, residencia del monarca, lugar de esparcimiento o finca de reposo- estaban enclavados en lugares de singular belleza y riqueza faunística o forestal. Por otra parte, no sólo las cualidades del agua mineral, sino también las del emplazamiento en un paisaje singular, han dado lugar al nacimiento y pervivencia de los balnearios, y a ello debe añadirse una buena relación con el exterior. La calidad de sus aguas, la belleza del lugar, la proximidad a la Corte $(122 \mathrm{~km}$.), el gran potencial de clientela que esto proporcionaba y el pertenecer al conjunto de propiedades que constituían el Patrimonio inmueble de la Corona hicieron de los antiguos Baños de Sacedón el lugar elegido para edificar una nueva población. Ésta había de convertirse en el Real Sitio a la medida de unas nuevas costumbres y de un estilo de vida adoptado por los monarcas a comienzos del siglo XIX.

\footnotetext{
5 Véase en Termalismo antiguo. Actas del I Congreso Peninsular celebrado en Arnedillo 3-5 octubre, 1996 (M. ${ }^{a}$ Jesús PEREx Agorreta, ed., Madrid, 1997): :Termalismo antiguo en los balnearios del siglo $x I X$ ", intervención de Juan José MOLINA VILLAR (págs.77-84), y también "Baños termales y fuentes medicinales en la Edad Media" (Capitulo VII).

6 Juan GayAn y SANTOYA: Antorcha metódica, mapa historial y discursos analyticos de las termales aguas de Sacedón, Córcoles, Trillo y Buendia. Madrid, Imp. de Gabriel Ramírez, 1758.
} 


\section{LA NUEVA POBLACIÓN DE LA ISABELA}

En otros Reales Sitios (Aranjuez, San Fernando, San Carlos en la Isla de León) ${ }^{7}$ se había construido anteriormente de nueva planta empleando el trazado en damero, logrando la armonía mediante la uniformidad de las fachadas y mediante la regularidad de sus vías. El principal hecho diferenciador viene marcado por el destino previsto para cada lugar, que determina su tamaño y el protagonismo del palacio y de las instalaciones, y que justifica la creación de cada Real Sitio.

Hay que situar también los precedentes de la configuración urbanística de La Isabela en la tradición fundacional hispánica, donde el plano ortogonal aparece constantemente, al igual que en la gran empresa colonizadora de Hispanoamérica, más cercana en el tiempo ${ }^{8}$, con una sugerente conexión política que relaciona ambos procesos: el de colonización interior y el de aseritamientos en las colonias de Hispanoamérica, como extensión del dominio de la Corona hacia nuevos territorios.

Este modelo en damero se adapta bien a un caserío que, por su tamaño y por su ubicación, no se prestaba a encuadres ampulosos, haciéndonos ver que la grandeza no se mide sólo por la magnitud de las construcciones, sino que puede servir de referente otra escala de valores sustentada en los principios de sobriedad y eficacia.

La Isabela añade a este planteamiento el concepto de urbanismo aplicado al campo ensayado, por ejemplo, en los núcleos rurales diseminados por diversas fincas de Aranjuez. Recordemos que Fernando VI puso en marcha la renovación urbanística de los Sitios Reales, pero fue Carlos III quien urbanizó en ellos los pequeños poblados que, generalmente, se habían formado previamente al azar. Surgió entonces el planteamiento de transferir a la edificación rústica en el entorno de los Sitios Reales las ideas cultas y de prestigio $^{5}$. Fueron la regularidad y la geometría recursos de que se sirvieron los arquitectos de la llustración para imponer un nuevo orden estético en el ámbito rural, criterio divergente al del urbanismo vernáculo ${ }^{10}$, del cual supieron apreciar las construcciones sobrias y eficaces. Haber aunado en ella estos criterios convierte La Isabela en ejemplo de organización arquitectónica rural.

Jordi Oliveras: Nuevas Poblaciones en la España de la llustración. Barcelona, 1998

- Agulleta Rojas, J., ibanez montoya, J., Moreno Rexach, Luis J: Urbanismo español en América. Madrid, 1979. En uno de los apartados recoge proyectos fechados ente 1700 y 1810 cuando, una vez estabilizada la colonizacion, se acentúa el crecimiento.

9 Virginia TOVAR: "Arquitectura suburbana en los Sitios Reales (siglo XviI)". Jornadas sobre arquitectura popular en España, 1987. Madrid, CSIC, 1990.

10 Julio CARo Baroja. (dir.): Arquitectura popular en España, Madrid, 1990. 

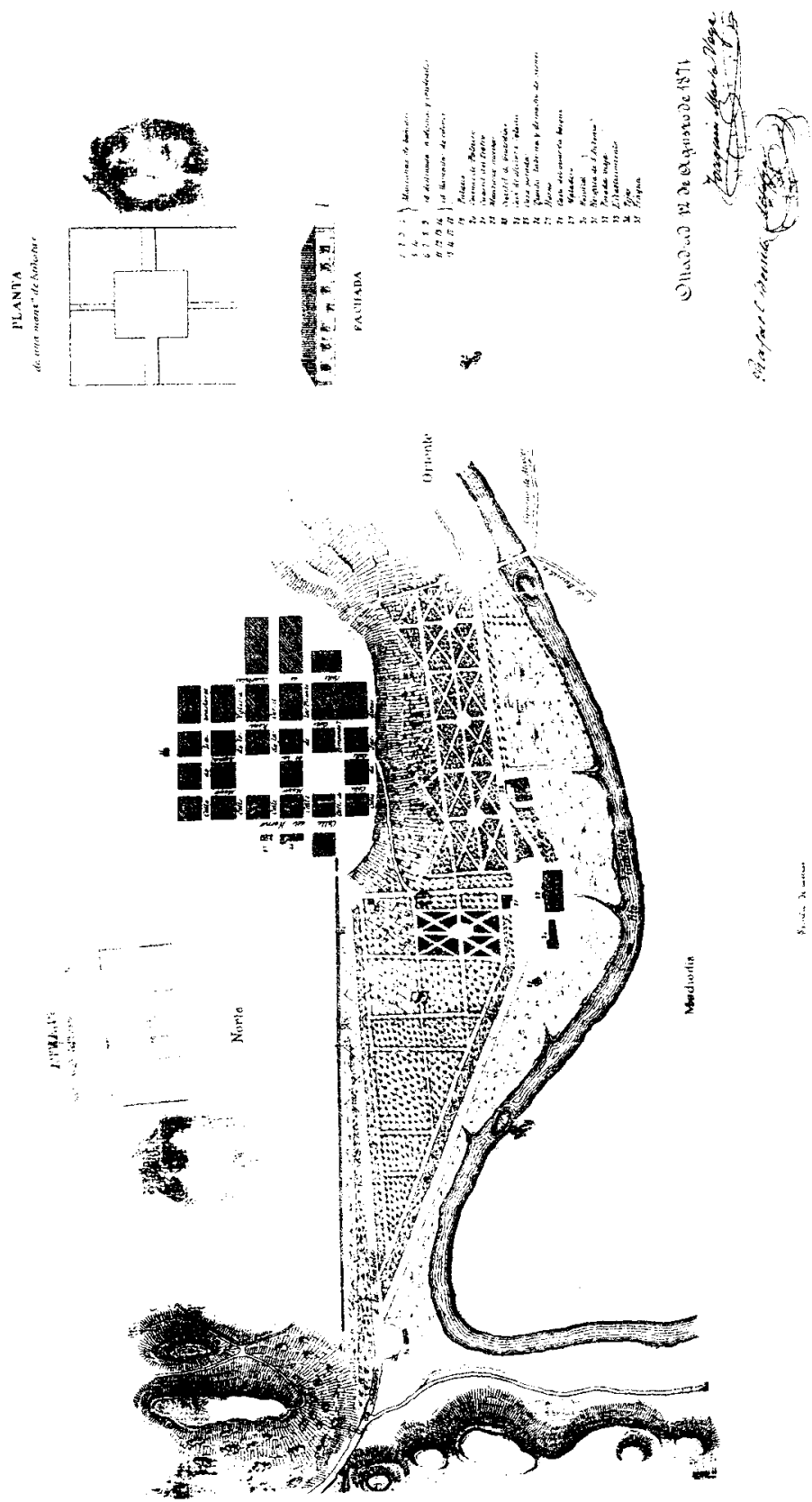

Fig. 2. La nueva población de La Isabela. Archivo Histórico Provincial de Guadalajara. Hacienda. Desamortización, 342. 
El arquitecto elegido para proyectar la nueva población que surgiría junto a los Baños de Sacedón fue Antonio López Aguado. Desde agosto de 1813 hasta julio de 1814 había asistido a las obras en los palacios y posesiones reales en calidad de Arquitecto Mayor, pero fue cesado en sus funciones ${ }^{11}$ por haber sido nombrado otro, Isidro Velázquez. El encargo de este proyecto a López Aguado suponía, sin embargo, un reconocimiento hacia él por parte de la Real Casa.

Las obras comenzaron oficialmente en enero de 1818 y progresaron rápidamente. La distribución de los edificios en La Isabela se realizó en 11 calles rectas, tiradas a cordel y dos plazas: Plaza Mayor y Plaza de la Constitución, llamada también del Mercado. El tamaño del caserío no impidió dar cabida a esta segunda plaza, diferenciando núcleos de actividad, tal como sucedia en otras nuevas poblaciones y Reales Sitios.

A pesar de la regularidad del trazado, éste no era simétrico: las manzanas del otro lado de la calle de Labradores por el norte, las situadas al otro lado de la calle del Horno por el oeste y la manzana 20 por el este rompían ligeramente la simetría. La ubicación del palacio en un extremo de la población, donde se obtenían las mejores vistas, no impidió que desplazara el protagonismo que la plaza mayor adquiría en otras nuevas poblaciones con este trazado, puesto que aquel podía verse desde que se entraba en la población.

En el plano observamos la existencia de 27 manzanas de edificios cuadrados, casi todos ellos de cien pies castellanos por cada lado, y de un piso, a excepción del Real Palacio. Las manzanas situadas entre la Calle Real y la Calle de Cuarteles son rectangulares, adaptándose a las dimensiones del perfil lateral del palacio (manzana 19). Dos de ellas, las más alejadas de él, pensadas en principio para alojar caballeria e infantería, se acabaron destinando a viviendas de colonos. La número 23, más próxima a palacio, era el Cuartel de Guardias. A continuación de él, la manzana 24, concebida originariamente como casa de oficios, acabó siendo destinada en parte a iglesia parroquial y el resto a habitaciones para bañistas. La Manzana 20, justo detrás del palacio, albergaba las cocinas. En su misma calle, la manzana 21 se destinó a teatro. Era un gran salón donde también

11 No obstante, López Aguado ambicionaba el cargo y esgrimió sus méritos para acceder a él en varios escritos dirigidos al Rey y a la Reina durante los años 1818 y 1819 . En ellos hacia presente que se le había encargado dirigir las obras de los reales Baños de Sacedón, casa-palacio y la nueva población denominada La Isabela, como asimismo el nuevo coliseo de la Real Plaza de Oriente, y el Casino y jardín de recreo de la Reina. Finalmente se aceptó satisfacerle con una gratificación en concepto del tiempo que tuvo a su cargo la dirección de las obras expresadas, pero no el nombramiento que solicitaba. (Archivo General de Palacio, Exp. personal, C. ${ }^{a} 559 / 19$ ). 
se organizaron bailes. Como criterio, las manzanas situadas en el ángulo exterior mejor aireado y con mejor panorámica, se destinaban a alojar a los bañistas. Las situadas céntricamente, entre la Plaza Mayor y la Plaza del Mercado, se destinaban a oficinas y viviendas de empleados de la Administración Patrimonial. Las demás constituian las viviendas de colonos.

La distribución habitable del hogar respondía a un estado social por encima de una moda o un gusto. Las viviendas eran edificios eminentemente prácticos que respondían a las necesidades básicas de sus moradores, ya se tratase de colonos, de bañistas o de personal de la Administración. Eran casas con sencilla fachada de una planta, blanqueadas en el exterior, con puertas y ventanas pintadas de verde. Las casas ocupadas por colonos fueron concebidas para funciones agropecuarias y como residencia. Constaban de: portal, sala con alcoba, otra sala o alcoba en el lado opuesto, una cocina grande y un cuarto frente a ella, patio o corral con cobertizo, cuadra, pajar, cámaras para los granos, y bodegas o cuevas en algunos casos.

Las viviendas dispuestas para bañistas eran de cuatro clases: superior, primera, segunda y tercera clase. Las antiguas tenian ocho cuartos cada una (cuatro de primera y cuatro de segunda), la manzana nueva catorce (cuatro de primera y diez de segunda), en la casa de oficios había trece (tres de primera y diez de segunda), dieciocho en el cuartel de guardias (dieciséis de primera y dos de segunda). Las manzanas antiguas, divididas en ocho habitaciones cada una, tenían cuatro portales que se cruzaban y reunian en un patio general, espacioso y bien empedrado, al que daban las ventanas de las cocinas. Estos portales semejaban una galería que se convertía en punto de reunión de los vecinos.

El buen tono hacía que se prefiriera vivir en las manzanas del Sitio y no en las habitaciones del Establecimiento de Baños. Éstas solían ser ocupadas por los muchos manchegos y labriegos que acudian a los baños particularmente a principio y fin de temporada, y también por personas impedidas, a fin de evitar los desplazamientos entre la población y los baños.

Al exterior de la Calle del Horno, en la periferia, frente a la huerta, tres manzanas ofrecian a la población los servicios de posada, tienda-taberna y horno. También en la periferia, pero entre el área de manzanas de colonos y los cuarteles, estaba la herrería.

A las afueras, la casa del guardabosque y el tejar se hallaban más apartados debido, en el primer caso, a la necesidad de ubicarla en el lugar que había de vigilar y, en el segundo, para evitar a los vecinos las molestias que ocasionarian los humos. Situado a la entrada por el camino de Sacedón, el tejar constaba de dos hornos destinados a la elaboración de 
materiales para las obras del Real Sitio. Aproximadamente medio kilómetro al norte de la población estaban el pozo de la nieve y el camposanto, respondiendo a criterios de salubridad, implantados en reinados anteriores en los Reales Sitios.

Un pequeño núcleo de construcciones, también apartado de la población, situado entre los jardines y el río, estaba constituido por el establecimiento balneario, matadero, hospital, ermita y posada vieja. En este área, en el entorno inmediato a los Baños, era donde se asentaban temporalmente los bañistas y quienes les abastecian antes de que fuera construida la nueva población. Ello motivó que el Infante don Antonio decidiese edificar allí la ermita, dedicada al patrón, San Antonio de Padua, y que se hubiera construido la posada vieja. Es también lógico que allí, junto a los Baños, estuviese el hospital. Situado al oeste de la Casa de Baños, se destinó a militares y pobres de solemnidad. Era un pequeño albergue con una sala grande de enfermería y otras tres piezas pequeñas.

A diferencia del planteamiento en El Escorial, La Granja o Aranjuez, la arquitectura de Antonio López Aguado en La Isabela, condicionada por la inestabilidad política y por las limitaciones económicas de la Administración Patrimonial, debía cumplir una finalidad eminentemente práctica: alojar a la familia real durante una breve estancia en un núcleo de población que había de ofrecer habitaciones y viviendas adecuadas a bañistas de diversa condición durante la temporada de baños, y un modo de vida digno a los colonos, cuyas viviendas no podían desmerecer del cercano palacio.

Originariamente no se planteó que transcurrieran las jornadas de la familia real junto al Establecimiento balneario, fue tardía la decisión de construir allí el palacio, que había comenzado a edificarse en la cercana villa de Sacedón ${ }^{12}$. En el de la nueva población empleó López Aguado un lenguaje estético sencillo con racionada geometría. Utilizó la composición bicrómica, tan característica de nuestra arquitectura, combinando sillares de piedra caliza en la parte inferior y ladrillo, según un estilo adoptado desde los Austrias ${ }^{13}$ con preferencia en lugares campestres como este palacio, de dimensiones intermedias y adaptado a las funciones de descanso y de relación con la naturaleza.

12 En la Memoria sobre el origen y estado de los Baños (Archivo General de Palacio, Isabela y Sacedón, 10870/16), además de recordar que el Consejo de Castilla declaró Real Sitio los baños por RI. Cédula expedida en 1802, se dice que en 1816 el Rey mandó suspender la obra de la casa-palacio que se estaba construyendo en Sacedón para que esa asignación se destinase a hacer nuevos los baños

13 Fernando CHECA y Miguel Moran: Las casas del Rey. Casas de campo, cazaderos y jardines. Siglos XVI y XviI. Madrid, 1986. 
La fachada principal miraba a Occidente y constaba de 12 ventanas y 13 balcones. El de enmedio ocupaba el ancho de la puerta principal sobre la que estribaba. Encima de los ángulos de la fachada había dos torreoncillos rebajados con un hueco a cada frontis del ángulo exterior. En las cuatro esquinas de la fachada y en los costados de la puerta principal existían grandes guardacantones de piedra caliza.

Era un edificio de dos pisos. La planta baja constaba de 37 piezas y un cuarto para excusados. La principal de 31 piezas. La planta baja tenía dos ingresos en los centros de la fachada principal y posterior. Destacaba en el interior la amplia escalera imperial, de tres tiros embarcando con uno central y volviendo con otros dos laterales. Respondía al tipo de palacio que estructura su espacio en torno a un gran patio central en el cual se sitúan las diferentes zonas. Tenía galerías de circulación en todo su contorno, con dos crujias paralelas a cada una de las fachadas laterales y una sola paralela a cada una de la principal y la posterior. Dentro de su austeridad, síntesis entre la imagen de palacio en el campo y el ideal de vida en la naturaleza, reunía características para ser morada de reyes, aunque no llegó a serlo puesto que las jornadas reales dejaron de tener como destino los Baños de Sacedón en $1826^{14}$.

La población se elevaba en un montículo sobre el río. En su base, al pie del palacio, adaptándose al terreno y en dirección al Establecimiento de baños, se extendían los jardines, de trazado geométrico. Debió marcar la predilección por éste la influencia de los proyectos para jardines que venían realizándose en nuestro pais, pero es también posible que fuera considerado más acorde con la sobriedad del trazado urbano y de las construcciones. Pese a la proximidad de las plantaciones de árboles y de la franja de bosque, estos jardines no aparecen vinculados al concepto de jardín romántico, puesto que éste se concebía creando ciertos efectos psicológicos. Así, el jardín paisajista se dividía a través de un recorrido sinuoso, estableciendo diferentes puntos de vista y proponiendo distintas escenografias. Otra de las características del prototipo de jardín pintoresco es la intimidad ${ }^{15}$, difícilmente compatible con el uso público, como lugar de ocio y de relación, a que en La Isabela se destinaba.

Los casos paralelos en el tiempo que cabe citar de creación de jardines en Reales Sitios son el Casino de la Reina y Vista Alegre. Ambos respondían

14 Archivo General de Palacio, Administrativa, C. ${ }^{\text {a }}$ 259/68.

15 V. Soto CABA destaca los reservados, lugares de recreo para la Familia Real que fueron acotados durante el reinado de Fernando VII, como los primeros jardines paisajistas de Madrid tras las experiencias de los Osuna y de Aranjuez (Jardines de la llustración y del Romanticismo en Es. paña. Madrid, 1993). 
a otro modelo: estaban surcados por calles sinuosas, donde las praderitas convivían con arbolado y matorrales espontáneos. Pero los tres jardines mantenían algo en común: aunque se trataba primordialmente de Sitios de recreo, esto se compaginaba con el cultivo de cereales, frutales, viñedos y otros productos. $Y$ es que un aspecto de la tradición jardinera castellana que supo mantenerse era la del huerto-jardín, donde armonizaban arte y utilidad.

La huerta y jardines de La Isabela, además de su faceta productiva, contribuian al pintoresquismo de la población y a engrandecerla mediante la perspectiva: una de las calles que bordeaban la huerta tenía como fondo el palacio, otra desembocaba en el Establecimiento. El paseo, continuación de la calle de Fernando VII que partía desde el palacio, dejaba a su izquierda los jardines reales y a su derecha la huerta del patrimonio, finca tan abundante en aguas que podía permitirse tener varios estanques y fuentecillas. La extensión de su huerta y jardines, y su enclave convertian la población en un lugar lo bastante ameno y apacible para convertirse en uno de los "espacios del poder» ${ }^{16}$. Por otra parte, en La Isabela el jardín era, a la vez que prolongación de las estancias palaciegas, lugar de relajo y deleite de los bañistas, estableciendo con ambas facetas relación entre el desarrollo de los parques y los jardines en el urbanismo de balnearios de la segunda mitad del siglo XIX y una concepción estética de la vida similar a la que había inspirado anteriormente los jardines de los Sitios Reales ${ }^{17}$.

El edificio que adquiere mayor protagonismo, núcleo vital de la nueva población, es el Establecimiento de Baños. Visitados a lo largo de siglos, es ahora cuando los Baños de Sacedón son objeto de la remodelación que los dignificaría. No llegarían a ser dotados de la grandiosidad y el fasto de los más afamados balnearios europeos del momento, pero, tomando como referencia las austeras casas de baños que por entonces existían en nuestro país, hemos de valorarlo como hito en nuestra arquitectura balnearia ${ }^{18}$.

16 Ana Vela y Luis A. Sanz consideran unido el origen de los sitios reales a una finalidad de cazadero real, entre otras funciones como la explotación agricola y ganadera, aprovechamientos forestales, lugar de esparcimiento o reposo. Todos, independientemente de su función estaban enclavados en bellos lugares ( «Los reales sitios: El espacio del poder». Alfoz, 1983, n. 4, págs. 73 76).

"La segunda mitad del siglo pasado desarrolló en el urbanismo de los balnearios la creación de parques y jardines que tuvieron su fundamento no sólo en la vida social que acogían, sino en una concepción estética de la vida, hasta el punto de hacerse consustanciales con ellos. Quizá en su origen pudiera verse una copia de los jardines de los Sitios Reales" (Luis FERNANDEZ FUSTER: Historia general del turismo de masas. Madrid, 1991, pág. 127).

18 Real Academia de Bellas Artes de San Fernando, 30-6/2: "Casas de campo y casas de baños 1800-1854" 
El Establecimiento balneario ${ }^{19}$ se encontraba a las afueras de la población, ante una plaza grande y rodeada de árboles muy próxima al Guadiela, donde la surgencia acuífera dio lugar a la creación de los antiguos Baños. Formaba un cuadrilátero con alzado de gran sobriedad en el que sólo aparecía resaltada la puerta principal por medio de cuatro pilastras con sus pedestales corridos y cornisa, componiendo un orden completo. Constaba de dos pisos, conocimientos de piedra y el resto en ladrillo.

Sus dos patios proporcionaban luz y ventilación. Uno se situaba en la parte posterior, donde se hallaban las cocinas. El vestíbulo comunicaba los dos patios, dispuestos con galeria cubierta, sostenida por pilastras de piedra asperón, material también utilizado en el estanque. A estas galerías daban los baños y los accesos a la planta principal.

En el ala izquierda existían una sala de reunión y un vestíbulo que daba al patio, un dormitorio junto al cuarto del encargado, una habitación de dos piezas, otra de cuatro piezas, una pieza de paso entre las dos antecámaras a las que se accedía por la entrada trasera, seis baños individuales con su cuarto y dos pequeñas piezas para comunes. En el ala derecha, una antesala comunicaba el vestíbulo con el patio por el que se accedía a los 14 baños individuales, al cuarto de un bañero, al de servicio $y$, mediante una pieza de paso a los tres habitaciones con baño. En el eje central, vestíbulo, estanque y dos baños colectivos, uno para cada sexo, a los que se accedía desde la pieza de espera por la puerta trasera.

El piso principal se hallaba distribuido de manera que un pasillo comunicaba los dos patios. En el ala izquierda habia cuatro cuartos de bañistas con tres piezas, dos con dos piezas, una pieza destinada a comunes y la azotea de servicio de los bañistas. En el ala derecha, cuatro cuartos de una pieza, cuatro de tres piezas, uno de dos piezas y la azotea de servicio.

En la parte central del edificio, la bóveda que cubria los baños colectivos ocupaba la altura de ambos pisos. Su armadura superior apoyaba en ambas fachadas, dejando un amplio desván. En el centro de la casa se encontraba el manantial formando un cuadrilátero de piedra asperón de 17 pies de largo, 12 de ancho y 5 de profundidad con un andito alrededor de su barandilla de hierro.

Como la ingestión de agua también tenía en los baños de Sacedón aplicación terapéutica, espacios comunes de acceso en la planta baja se destinaban a bebedor. Junto a la puerta principal del patio de la derecha

\footnotetext{
Archivo Histórico Provincial de Guadalajara, Hacienda, Desamortización, 14.107.
} 
se hallaba una pieza casi del todo oscura donde estaba la fuente para beber las aguas.

Pero en La Isabela la concepción arquitectónica del balneario transcendía a la simple organización de un edificio, es decir, parte del caserío se destinaba a alojamiento de los bañistas, el palacio fue edificado con el fin de que la familia real acudiera a los baños y los jardines se convertían en una alternativa de ocio, complemento a la terapia. De este modo, siendo el Establecimiento el eje en torno al cual giraba la vida de la población, buena parte de los edificios, aun alejados, constituian una prolongación de él. El hecho de que toda una población se concibiera en función de la existencia del balneario, bajo un concepto impregnado de talante filantrópico ilustrado, y la presencia de elementos, como es el palacio, de especial interés hacen que el conjunto destaque dentro de nuestra arquitectura balnearia. Balnearios como los de Trillo ${ }^{20}$, Arnedillo ${ }^{21}$ y Solán de Cabras ${ }^{22}$, que muestran cierto paralelismo con La Isabela por su origen en algunos casos y por su proximidad en otros, son un buen referente sobre la tendencia de esta tipología de construcciones.

La Isabela se caracteriza, además, por la peculiaridad de haber sido construida en un solo reinado, habiendo dado fin un mismo arquitecto a un conjunto organizado - lo cual rara vez sucede- y en un período de tiempo breve - poco más de una década-. Por ello refleja con claridad, tanto en la planificación como en los usos, la concepción de la sociedad en un momento preciso. Su importancia radica en ser último proyecto de envergadura de una nueva población, ejemplo de urbanismo ilustrado en un Real Sitio semejante a los emprendidos en tiempos de Carlos III, pero en una realidad política bien distinta y adversa a los intereses de una proporción considerable del Real Patrimonio inmueble.

Se convirtió en un lugar de asueto para el monarca donde resultaría fácil llevar una vida distendida alejada del aparato cortesano y en lugar de ocio para cualquiera que alli acudiese, donde la faceta más elitista quedaba marginada. Constituía a su vez una obra benéfica y un lugar donde se desarrollaban actividades económicas que contribuian a su sostenimiento. Una de ellas, la agricultura, era tradicional en el ámbito de los Reales Sitios, otra, la explotación de las aguas termales, suponía una novedosa

M. Esperanza Garcia Romo et al:: «El Baineario de Carlos Ill en Trillo". Wad-al Hayara n. 19 Juan Manuel López AzCona: Estudio sobre los baños de Arnedillo. Madrid, 1988.

22 Archivo General de Palacio, Solán de Cabras, C. ${ }^{3}$ 10421/31. Ver también Cadenas y V CENT, V.: Jornada de Fernando VII y Amalia de Sajonia en los Reales baños de Solán de Cabras en busca del deseado sucesor. Madrid, Hidalguía, 1957. 
incursión por parte del Real Patrimonio en un nuevo sector económico y, precisamente, en una faceta donde los empresarios de nuestro país, pese a contar con óptimos recursos naturales, no se permitían realizar inversiones cuantiosas.

\section{ORIGEN Y EVOLUCIÓN DEL REAL SITIO}

En origen, el balneario no era más que una piscina o una pileta al aire libre. En nuestro ámbito, permaneció como tal hasta tiempos muy recientes, y así se mantuvieron los Baños de Sacedón ${ }^{23}$. Las clases populares mantenían la tradición de acudir a estos lugares en busca de mejorar la salud. Los estratos sociales superiores, recuperaron esta práctica ${ }^{24}$ confiando en las virtudes que los nuevos tratados médicos atribuían al termalismo.

De ese interés renovado y de la estancia allí de doña Mariana de Austria nació la iniciativa por parte de la madre de Carlos II, de construir una casa próxima a estos Baños de Sacedón, situados en la zona denominada Dehesa de las Pozas. Ello motivó la donación en 1670 por parte de Huete del lugar donde se ubicaban los Baños a Carlos II y la intervención regia en las primeras obras para construcción de una casa ${ }^{25}$ próxima a ellos, cuya obra se paralizó a poco de empezada y fue continuada por don Pedro Núñez de Guzmán, Marqués de Montealegre, quien también halló en este lugar la salud. Pero todo quedó de nuevo arruinado a los 50 años.

23 "Manan estas aguas variamente por todo el suelo, subiendo con impetú hasta la superficie, aunque está llena la caxa, y entonces nadie puede hazer pie". Juan de la TORRE y VALCARCEL describe así el manantial en la obra de Fernando INFANTE Teatro de la salud, baños de Sacedón..., editada en 1663 y dedicada al Marqués de Montealegre, que reedificó los Baños. Adjunta un croquis ubicando Sacedón con respecto a Madrid y Aranjuez y representando "la fábrica que hizo el Sr. Marques de Montealegre".

24 Gayan y Santoyo en Antorcha Metódica., mapa historial y discursos analyticos de las termales aguas de Sacedón, Córcoles, Trillo y Buendia (Madrid, Imprenta de Gabriel Ramirez, 1758) propone "para diversión de los lectores" un relato basado en un manuscrito que dice estar en su poder, según el cual un rústico pastor. Phelipe Vengala, recomendó tomar las aguas a Gonzalo Fernández de Córdoba, que padecia dolores de reúma, cuando se hallaba desterrado en el castillo de Santaver o Cañaver. Al parecer, logró mejorar y difundió el hecho entre magnates de la Corte, que empezaron a mirar con interés aquellas aguas. Añade que en 1600 la tomó don Bernardo de Rojas y Sandoval, Arzobispo de Toledo, y en 1666 la Reina madre quien, en agradecimiento mandó se principiara una obra que, llegado el caso, sirviese de palacio a un rey y hospedaje a muchos necesitados.

25 Archivo General de Palacio. Isabela y Sacedón. C. 10870/16: "Memoria sobre el origen y estado de los Baños de Sacedón y Sitio de La Isabela. Y el oficio original de las Cortes al Ministro de la Gobernación en fecha 24 de junio de 1821 para que estos baños se administren por cuenta de su Majestad". 
Reactivado el fenómeno balneario en el siglo XVIII, cuando se despertó nuevamente el interés por esta terapia por parte de miembros de la familia real y de la aristocracia quienes, buscando un remedio confortable a sus dolencias, mejoraron las condiciones de acceso del resto de la población, como acto caritativo. Los balnearios se convirtieron en un recinto cerrado en el que aparecieron otras funciones, donde convivieron tratamiento, albergue y ocio. Esta fase se inició en los Baños de Sacedón cuando el Infante don Antonio, hijo de Carlos III, empezó a acudir con asiduidad y ordenó realizar un estudio sobre las cualidades de sus aguas ${ }^{26}$. Visitó Los Baños en 1791 y, sorprendido por la lamentable impresión que le produjo su estado, promovió que desde entonces se fueran realizando sucesivas mejoras en ellos. Hizo reparar el manantial, recomponer la antigua casa y edificar la capillita de San Antonio.

Tuvo así continuidad la actuación de Carlos III, que culminó con la construcción de los baños de Trillo, en lo que podría considerarse un aspecto de la política sanitaria de Carlos IV, quien aportó los primeros fondos para rehabilitar los Baños. Fernando VII fue innovador al insertar en el modelo económico y de relaciones sociales dominante en nuestros balnearios una nueva población que complementaría ese modelo. Yuxtaponiendo a este planteamiento el de la colonización agrícola en la línea de las fundaciones reales de nueva planta, surgió la idea de crear junto a los antiguos Baños de Sacedón, cuyo establecimiento sería notablemente mejorado, una nueva población: La Isabela.

Pese a que pueda ser considerada continuación de la política repobladora, formando parte de un proceso amplio con origen en la Edad Media y dilatada experiencia en la América hispana, no consta que fuese La Isabela objeto de planificación a medio plazo. Tanto la acotación de su término ${ }^{27}$ como el proceso de construcción ponen de relieve conflictos que era preciso solventar sobre la marcha. Conflictos que nos remiten a la inestable situación de poder de la Monarquía y a la coincidencia de circunstancias de orden económico y social que, si bien justifican su creación, también explican el progresivo declive del Real Sitio.

26. Los problemas de salud que le aquejaban le llevaron a buscar remedio en el termalismo. En la decisión de acudir a los Baños de Sacedón es posible que influyera la lectura de la obra de Marino PIzzI Tratado de las aguas medicinales de Salam-bir que comunmente llaman Sacedón...,que se encuentra en la biblioteca del Palacio Real de Madrid. Del interés del Infante por que existiera un informe científico donde se aplicasen los conocimientos de la época es prueba el hecho de que mandara editar el Análisis de las aguas minerales y termales de Sacedón que se hizo cuando paso a tomarlas el Serenísimo Sr. Intante D. Antonio en el mes de julio y agosto de 1800 en 1801, cuadernillo que mandó imprimir a su costa en Madrid, en 1801 en la imprenta de Villalpando.

27 Libro de Apeos del Real Sitio de La /sabela. Archivo Provincial de Guadalajara, sig. 14.106. 
A las realizaciones llevadas a cabo por arquitectos de la importancia de Villanueva en las primeras pequeñas obras y de Antonio López Aguado en la nueva población han de sumarse proyectos de categoría, apetecibles para los grandes arquitectos, que nunca se hicieron realidad. Es decir, en la historia de La Isabela hay un apartado de realidades y otro de proyectos, diferenciados en tres fases:

I. La primera entre 1801 y 1816 cuando se hicieron pequeñas obras para habilitar los antiguos baños y una casa-palacio en Sacedón. Fue cuando, por motivos de salud, realizó asiduas visitas el Infante don Antonio, acompañado en los últimos años por Fernando VII.

El Consejo dio comisión en $1787{ }^{28}$ para la construcción de obras necesarias en los Baños de Sacedón y se aprobaron las ordenanzas formadas por el arquitecto Juan de Villanueva, quien realizó cierta obra en la que se reparó lo más preciso ${ }^{29}$.

II. La segunda, ya afianzado en el poder el Monarca, cuando transcurren allí algunas de las jornadas reales ${ }^{30}$, abarca desde 1817 hasta 1826. Es cuando proyecta Antonio López Aguado y se construye la nueva población, a excepción de la iglesia, algunos cuarteles, la casa de oficios y la casa de la Administración, que nunca llegaron a edificarse.

El 28 de enero de 1818 se puso la primera piedra. En seis meses estuvieron concluidas las dos primeras manzanas de casas. Sin embargo, no era el mejor momento para emprender un proyecto constructivo de tal magnitud. En la Gaceta de 24 de junio de 1819 se expide una Real órden a propósito de lo que ya había comunicado en Diciembre de 1817 el Secretario de Estado y del Despacho de Gracia y Justicia: el lastimoso estado a que se hallaba reducida la renta de Correos. En anteriores reinados se sufragaban con ella obras de primera magnitud, caminos, establecimientos científicos, escuelas, pensiones... pero sólo alcanzaba a satisfacer algunas deudas cuando, después de ocho años de guerra, era preciso reparar caminos, paradas de postas, posadas... y el ramo de Correos se prestaba a auxiliar a la real Hacienda en situación de grandes apuros.

28 Se remitió al Secretario de Cámara un expediente de 1787 sobre construcción de las obras necesarias en los Baños de Sacedón (Archivo Histórico Provincial de Guadalajara, sig. 14.107).

29 En documento fechado el 1 de agosto de 1801 se indica que “...en diferentes ocasiones se han dado providencias al insinuado fin por el RI. y Sppmo. Consejo de Castilla, pero no han producido el efecto deseado, y hace pocos años qe. el Arquitecto Mayor del Rey Ntro. Sr. hizo por su quenta cierta obra, pero se reduxo a lo más preciso... (Archivo General de Palacio, Isabela y Sacedón, C. a $10870 / 1)$.

${ }^{30}$ Archivo General de Palacio, Administrativa, Legs. 787, 788, 789, C. ${ }^{\text {a } 259 / 68 .}$ 
Podemos deducir que justificaron la continuidad de las obras el uso que podía hacer del balneario el Monarca y su intención de plasmar el sello personal de su reinado, emulando algunas de las obras más admiradas de su dinastía, con la creación de un nuevo Real Sitio y de manifestar en la práctica la continuidad con los programas de su abuelo, Carlos III, en las políticas poblacionista y benéfica. Pero Fernando VII no pudo salvar la distancia que imponía la diferente situación de poder de la Monarquía, no podía movilizar los recursos con que contaron Felipe $V o$ Carlos III.

La cuestión del presupuesto era preocupante cuando en 1820 el López Aguado se planteaba qué obras podían suspenderse en La Isabela sin detrimento de lo hecho, ya que el Rey había ordenado que cesaran las obras en Palacio y demás posesiones reales mientras mejoraba la situación ${ }^{31}$. Sin embargo, dio permiso para que continuaran las que se ejecutaban en La Isabela ${ }^{32}$.

Dada esta situación, sólo se pudieron retomar las obras con mejor disponibilidad económica y visos de éxito en 1824, una vez restablecidos los poderes absolutos de Fernando VII e iniciada la represión política. Ese empeño por seguir adelante queda de manifiesto en una breve nota autógrafa ${ }^{33}$ del Rey dando el visto bueno a las necesidades de personal que el arquitecto demandaba en ese mismo año. Sin embargo, el hecho de que continuaran no prueba que la situación económica hubiese mejorado, más bien empeoraba. Ello posiblemente influyó en que los resultados se apartasen de la idea inicial, no tanto en lo relativo al trazado como en lo referente a calidad de los materiales y del trabajo.

En 1826 quedaron tomadas las aguas del Palacio, pero aún quedaban en él obras pendientes, así como la edificación de cuarteles para tropa, casa de oficios, casa de la Administración e iglesia. La adversa situación sociopolítica condicionó el hecho de que la construcción fuera digna, pero eminentemente práctica, marcando cierta diferencia con la categoría de los edificios de otros Reales Sitios, con el acierto de encontrar apropiado y adecuar el esencialismo formal a una arquitectura no específicamente monumental. Arquitectura que hubiera sido complementada más adelante con edificios que engrandecerían el proyecto inicial.

31 Archivo General de Palacio, Fernando VII, C. ${ }^{a}$ 240/7. 1820: “Expediente sobre la supresión de obras de Palacio y posesiones reales".

32 Archivo General de Palacio, Fernando VII, C. ${ }^{3}$ 375/221.820: "El Arquitecto Mayor pregun ta sobre continuar o no las obras de Sacedón..

33 Archivo General de Palacio, Isabela y Sacedón, C. ${ }^{a}$ 10870/27. 
La participación de Isidro Velázquez fue contemporánea a su labor en el cerramiento de la Plaza de la Armería y a la reforma del Teatro en el Pardo y de la Capilla del Cristo del Convento de los Capuchinos. La iglesia parroquial de La Isabela ${ }^{34}$ le fue encargada en 1826 . Hubiera resultado la edificación más valiosa ${ }^{35}$. Compartiendo con el conjunto urbanístico las características de sobriedad y funcionalismo, destacaría por su originalidad, aproximándose más a la tipología de construcciones en otros Reales Sitios. Lo planteó como edificio compacto de disposición centrada sobre una planta cuadrada. La monumentalidad de la fachada daría paso a la nave con dos capillas laterales, cubierta con una gran cúpula. De haberse construido, ésta pudo haber sido la última gran realización del arquitecto, inmediata al final de su actividad profesional hacia 1830.

III. La tercera fase comprende desde la última fecha hasta 1874, cuando comienza la venta de fincas, conforme a la ley de desamortización de 1865. Por entonces continúan las obras, pero se trata de obras de mantenimiento, no de mejora, ni de ampliación, pese a los interesantes proyectos encargados a algunos de los arquitectos de mayor renombre del siglo XIX. Prueba de que en los Baños de La Isabela la intervención del Monarca suponia a priori posibilidades de mejora a gran escala son los presentados para el Establecimiento de baños, ya en el reinado de Isabel II, por Custodio Moreno y por Narciso Pascual y Colomer.

Custodio Moreno, quien ocupó al cargo de Arquitecto Mayor al jubilarse Isidro Velázquez, firmó en 1843 un proyecto para construir un balneario en La Isabela ${ }^{36}$. Éste mantendría la estructura central del edificio, pero lo engrandecería con una glorieta de entrada que daría acceso a través de una zona ajardinada al Establecimiento de baños. A través de una de las entradas del pórtico se accedería a su vez a la pieza de baño de SS.MM.. EI proyecto ratifica la intención por parte de la Casa Real de dar un nuevo impulso a La Isabela como Sitio Real, intención frenada por circunstancias sociopolíticas que limitaban las posibilidades de la Administración de la Real Casa.

\footnotetext{
34 J. MORENO VILLA: «Planos inéditos de don Isidro Velázquez para una iglesia en La Isabela». Arquitectura, 1932, n. 155 , págs. 69-76.

35 Archivo General de Palacio, Planos: 501, Fachada principal. 499, Fachada posterior 496, Sección. 497, Planta del piso principal 498, Planta baja.

36 Archivo General de Palacio, Planos, 3484
} 
No corrió mejor suerte el proyecto para Casa de Baños realizado por Narciso Pascual y Colomer ${ }^{37}$, discípulo destacado de Custodio Moreno, que como él llegó a ser arquitecto Mayor de Palacio. El proyecto no respetaba de la construcción antigua más que la piscina del manantial que, rodeada por 26 baños y cuatro pilas, se convertía en un edificio de planta octogonal inscrito en otro con planta cuadrilateral y aislado de él mediante jardines.

Existe otro interesante proyecto ${ }^{38}$, sin firma ni fecha, en el que se recurre a la estructura circular, convirtiendo el manantial en corazón del edificio y distribuyendo el espacio en torno a él en tres círculos concéntricos: uno constituido por los baños, otro por el gran corredor que daba entrada a ellos y a su vez a las salas de descanso, cuartos de bañeros, habitaciones para impedidos, etc., que conformaban en círculo exterior. Cabe explicar la drástica reducción en el número de baños y de habitaciones con relación a los proyectos anteriores como un ensayo estético en el que lo conceptual, relacionado con lo recoleto del lugar y la ausencia de bullicio, primase sobre lo funcional, relacionado con el máximo aprovechamiento de un establecimiento al que se pretendía dar auge. O bien interpretarlo como un último intento de adecuación a las posibilidades del Establecimiento en función del número de bañistas que venía acudiendo a él.

Es esta arquitectura sobre el papel la que eleva los edificios de La Isabela a la categoría de los más grandiosos de otros Reales Sitios. Y es así porque las circunstancias sociopolíticas se impusieron al proyecto en el que creyó Fernando VII y que pretendió apoyar Isabel II, determinando esas circunstancias que el resultado en cuanto a calidad material y riqueza arquitectónica se refiere no estuviese a la altura, y que sucesivos proyectos de modernización y ampliación del Establecimiento balneario fueran fracasando. De ahí que otro aspecto interesante que revela La Isabela es el reflejo de "la otra cara" de los Reales Sitios: lo que queda del refinamiento de la villa cortesana concebida por los Borbones una vez pasado por el tamiz del cambio sociopolítico gestado a comienzos del siglo XIX.

Lo anterior nos conduce a valorar la viabilidad de este Real Sitio como nueva población, pervivencia en el siglo XIX de la política poblacionista ilustrada, y como balneario, para señalar diversos factores que contribuyeron a su decadencia: el deterioro material, las actitudes sociales, la trascendencia de la normativa legal, las mutaciones en la organización espacial y el potencial de La Isabela en cuanto a rentabilidad económica.

37 Plano sin fecha entre los pertenecientes a La Isabela (Archivo General de Palacio, Planos, 3483).

38 Archivo General de Palacio, Planos, 3485. 


\section{CONDICIONANTES DEL DETERIORO DEL CONJUNTO URBANO}

Las limitaciones impuestas a la Corona en el siglo XIX para disponer de recursos económicos se convirtió en el principal condicionante del devenir de La Isabela desde que se iniciaron las primeras obras de envergadura. Momentos críticos fueron la búsqueda de fondos por parte del Infante don Antonio para realizar las primeras obras en los Baños ${ }^{39}$, cuando el gobierno de Carlos IV se hallaba oprimido por los gastos de guerra y se producia un astronómico crecimiento de la deuda pública, y el Trienio liberal, cuando hubo de ser solicitado permiso a las Cortes para sufragar obras con fondos ya considerados públicos, y el permiso fue inicialmente denegado por no existir aclaración sobre la titularidad del propietario ${ }^{40}$.

Es preciso añadir que en La Isabela las obras de mantenimiento como consecuencia ya fuera de las continuas crecidas del río Guadiela, ya del apresuramiento en la construcción o de deficiencias del material, requirieron una continua remisión de fondos por parte de la Administración patrimonial de la Real Casa, fondos que de otra manera hubiesen podido revertir en los interesantes edificios proyectados y no construidos. Cuando a esto se unieron los cambios en la organización espacial y en el sistema de propiedad que estaban teniendo lugar, las nuevas actitudes sociales y la falta de eficacia de la normativa protectora, sucedió que, como bien ejemplifica el caso de La Isabela, a lo largo del siglo xIx el Patrimonio inmueble de la Corona, parte muy notable de nuestro legado actual, quedó mermado considerablemente.

Varios factores que cambiaron la organización espacial se sucedieron para condicionar su desaparición: en primer lugar uno de orden administrativo, la conversión de la nueva población en aldea pedánea de otro municipio, en segundo lugar otro que añade implicaciones socioeconómicas, la desamortización, y por último la necesidad de adaptación a la infraestructura de un país en desarrollo.

39 Ante las dificultades para financiar el proyecto, se propone cortar 70.000 pinos, o más, de Cuenca e inmediaciones. Se acepta la corta de 12.000 o 14.000 para adecentar el Establecimiento considerando que «No carece de exemplo este proyecto/.../pues con otro igual se hicieron las obras y casa de Solán de Cabras..." (Archivo General de Palacio, Isabela y Sacedón C. ${ }^{\text {at }}$ 10870/1: "Informe sobre la reconstrucción de los reales Baños. Se acompaña una propuesta del coste y beneficio que se podria sacar de cortar y llevar por el Tajo hasta Aranjuez 6.000 pinos, para lo que se pide autorización a S.M.»).

40 Archivo General de Palacio, Isabela y Sacedón, 10870/16: "Memoria sobre el origen y estado de los Baños". 
La Isabela se convirtió en 1840 en aldea pedánea de Sacedón, municipio que la consideraba popularmente como un barrio del que obtenía prestigio. A los habitantes de la nueva población no les satisfizo tal medida. Les parecía una injusticia que pueblos de la provincia con menos vecindario tuvieran ayuntamiento propio y pudieran entenderse con el gobierno directamente. En una carta a la Reina de 1845 se quejaban de la animosidad de los de Sacedón contra ellos ${ }^{41}$ y de su actitud que les impedía «... disfrutar de los beneficios que las leyes les conceden..." ${ }^{42}$. $Y$ es que, efectivamente, la nueva población y los isabelinos contaban con privilegios en lo referente a impuestos, ejército, reemplazos... que en los tiempos que corrían eran cada vez peor vistos: el Ayuntamiento constitucional de la vecina Sacedón se oponía abiertamente a los derechos patrimoniales ${ }^{43}$.

A esta actitud se suman los actos de vecinos de los alrededores y de facciosos contra las propiedades patrimoniales ${ }^{44}$ en tanto que constituían un emblema de la Monarquía. Por otra parte, los mismos colonos, desmotivados por las trabas para acceder a la propiedad de las colonias ${ }^{45}$, tendían a reparar sólo lo imprescindible para habitar su vivienda, y aún se sumaba la desidia de sucesivos administradores y la no ocupación de puestos laborales previstos en el reglamento ${ }^{46}$. Todos ellos son componentes sociales del condicionante local que contribuyó al deterioro del conjunto urbano y que contrarrestó el efecto positivo generado por otra actitud social: el fenómeno del termalismo en el siglo xIX. Efecto positivo hasta

41 Animosidad confirmada por el Interventor, que comunicaba en 1841 la oposición de los concejales de Sacedón a los derechos patrimoniales (Archivo General de Palacio, Isabela y Sacedón, C. ${ }^{\text {a } 10.878 / 32) . ~ P o c o ~ a n t e s ~ s e ~ h a b i ́ a ~ r e c i b i d o ~ l a ~ q u e j a ~ p o r ~ p a r t e ~ d e l ~ A l c a l d e ~ c o n s t i t u c i o n a l ~}$ de Sacedón de la oposición que hacían los vecinos de La Isabela al Ayuntamiento (Archivo General de palacio, Isabela y Sacedón, C. ${ }^{a} 10.878 / 23$ ).

42 El propio Ayuntamiento de Sacedón tenía dudas sobre si considerar a los colonos de $L a$ Isabela habitantes de Sacedón a efectos civiles. Pero consideraba injusto que la posada u otros edificios que daban beneficios no estuvieran sujetos a contribución y opinaba que el Alcalde debia atribuirse prerrogativas sobre administración del Real Sitio (Archivo General de Palacio, Isabela y Sacedón C. ' 10.882/14).

4.3 Libro copiador de las comunicaciones y consultas que por esta administración se dirigen a la General de la Real Casa y Patrimonio (Archivo Histórico Provincial de Guadalajara, Delegación de Hacienda, 13971).

${ }^{44}$ Archivo General de Palacio, Isabela y Sacedón, C. ${ }^{a} 10.873 / 11$, C. ${ }^{a} 10.876 / 10$, C. ${ }^{a} 10.877 / 2$.

45 El informe del Interventor sobre un expediente promovido en 1846 es drástico: «... si los colonos adquieren la propiedad del terreno y gran parte del caserío rehuirían los bañistas porque los propietarios tenderian a estafarlos, mientras que la Administración vela que los arriendos sean a precios arreglados". Es un argumento que no hace sino confirmar las características del enfrentamiento entre Administración y colonos. Aparecen en el expediente las escrituras otorgadas a los colonos en 1851 y el pliego de condiciones propuestas por la Contaduría General de la Real Casa para el arriendo por 25 años (Archivo Histórico Provincial de Guadalajara, 14.107).

46 Archivo General de Palacio, Isabela y Sacedón, C. ${ }^{a} 11823 / 8$. 
que la farmacopea moderna, la moda de los baños de mar, favorecida por la mejora de las comunicaciones, y el nuevo concepto de balneario, que conducia a la transformación de los establecimientos termales en grandes hoteles, convirtieron la configuración austera de La Isabela y su filosofía benéfica en algo obsoleto.

El terreno montuoso y los dos ríos: Tajo, y Guadiela, confluyentes en las cercanías de la población, limitaban y complicaban los accesos a ella. Distintos visitadores del Real Patrimonio achacaban la escasa afluencia en buena parte a un camino lleno de incomodidades. Mediada la década de 1860 la carretera Madrid-Cuenca que se estaba construyendo pudo haber sido desviada para que hubiese pasado más próxima al lugar, pero ello hubiese supuesto aumentar en unos diez kilómetros el trayecto entre ambas capitales. Así, La Isabela, en lugar de verse beneficiada por esta mejora en las vias de comunicación, se vio perjudicada porque, en los años que duraron las obras de esta carretera, buena parte del camino se encontraba obstruido.

La alternativa era el ferrocarril, impulsado por la Ley de 1855. En 1864 se presentó un proyecto de ferrocarril de Madrid a Reus por Cuenca, con parada en la vecina población de Huete, presentado como arteria económica que pondría a las puertas de Madrid productos de Cataluña, de Aragón y de las comarcas más ricas de Cuenca y parte de Guadalajara ${ }^{47}$. Pero las dificultades para desarrollar este tipo de infraestructura en un corto plazo y la paralización relativa que hacia 1866 sufrieron las obras ferroviarias como consecuencia de la crisis agraria y financiera dieron al traste con el potencial que pudo suponer la extensión del ferrocarril. El citado Castellanos de Losada llegó a afirmar que podría convertirse en el Versalles de Madrid durante los veranos. Sin embargo, los bañistas se trasladaban a balnearios que contaban con mejores accesos conforme en el último tercio de siglo progresaba el trazado de vías férreas ${ }^{48}$, aun cuando no fuese frecuente que esos otros balnearios poseyeran en un principio elementos que este Real Sitio tenía para ser un primer establecimiento en su género.

\footnotetext{
47 Ordónez: Memoria sobre el proyecto de ferrocarril de Madrid a Reus por Cuenca, Teruel, Utrillas, bajo Aragón y el Priorato. Madrid, 1864.

48 Chernoviz: Guía Médica compuesta de tres partes principales Formulario médico conteniendo la descripción de los medicamentos, su dosis, las entermedades en que se emplean y las mejores fórmulas. "l Compendio de las aguas minerales de España, Portugal, Francia, Bélgica. Suiza, Alemania y América meridional. Acompañado de vistas de algunos establecimientos ter. males y de 5 mapas balnearios. III Memorial terapéutico o descripción abreviada de los sintomas y del tratamiento de las enfermedades. París, A. Roger y F. Chernoviz, 1880.
} 
Entre 1864 y 1869 se produjeron cambios administrativos tendentes a la separación definitiva entre el patrimonio de la Corona, el patrimonio del Estado y el patrimonio privado del Rey, situación a que la que abocó el clima político. El progresivo declive de La Isabela se inició al verse afectada por el más radical de ellos, la Ley desamortizadora de $1865^{49}$. La recuperación del auténtico valor de la propiedad hubiese sido posible de no haberse rechazado la propuesta del Departamento de Sanidad del Ministerio de la Gobernación de quedarse con La Isabela por una parte de su valor de tasación para fundar un establecimiento balneario modelo ${ }^{50}$. Su resurgir dependía de una cuantiosa inversión pero, una vez desamortizada, faltó la continuidad de los propietarios ${ }^{51}$, apremiados por especular o por obtener beneficios inmediatos.

Su último propietario fue el Marqués de Vega Inclán, quien fuera Primer Comisario de Turismo ${ }^{52}$. La sensibilidad del Marqués, su carácter emprendedor, su visión en perspectiva a largo plazo, le hicieron percibir que el éxito de la rehabilitación de edificios se fundamentaba en motivos sociales y económicos aunados a los culturales, llevando a primer plano los intereses de la población residente, factor crítico en nuestro caso. Pero ello no bastó en este proyecto.

El progreso de la industria y la mejora en la calidad de vida motivaron que, ya concluidas las principales vías férreas, en la primera década del siglo xx España empezara a incorporarse a la electrificación. El pantano de Bolarque en el curso del Tajo a pocos kilómetros de la desembocadura del Guadiela, se construyó para suministrar energía a Madrid. Era el primer paso para que se elaboraran los proyectos de los pantanos de Entrepeñas y Buendía. Los tres formarian uno de los mayores complejos hidroeléctricos existentes en Europa. La propuesta del pantano de Buendía, cuya extensión comprendía el término de La Isabela, fue hecha en $1931^{53}$

49 Archivo Histórico Provincial de Guadalajara, Hacienda, Desamortización, 14.107.

50 Archivo General de Palacio. Administrativa, Leg. 538.

51 Registro de la Propiedad de Sacedón, Tomo 108.

52 Su éxito en la divulgación del Toledo artístico, su conocimiento de la vida y costumbres en el extranjero, su relación con Palacio, su acta de diputado y su carácter activo debieron ser los méritos que consideró el Presidente del Consejo, D. José Canalejas, para nombrarle Comisario de Turismo. Al frente de la Comisaría Regia desarrolló una gran labor creando los primeros paradores nacionales, restaurando el patio de yeso del Alcázar sevillano y el barrio de Santa Cruz y publicando la colección "El Arte en España". A él también se debió la Casa-Museo del Greco en Toledo, la Casa de Cervantes en Valladolid y el Museo Romántico en Madrid (Francisco Azorín: El Marqués de Vega Inclán y el Museo Romántico. Madrid, 1977. Vicente Traver: El Marquês de Vega-Inclán. Primer Comisario de Turismo y Cultura Artística Popular. Castellón, 1965).

53 Fecha del primer informe, aunque la Memoria fue concluida en 1941 (Archivo General de la Administración, Obras Públicas, Sig. 1.475, C. ${ }^{a} 14.757$, Leg. 18605). 
ante la necesidad de regular las crecidas del Guadiela, evitar riadas, aumentar el regadio, para un posible aprovechamiento de la industria y para producción de energía.

No se contemplaba esto en los planes del Marqués de Vega Inclán cuando, en 1930, adquirió los baños entendiendo que se trataba de «... uno de los dominios hidrológicos medicinales más interesantes y quizás de mayor porvenir en España” ${ }^{54}$. Tuvo, además, la mala fortuna de desplegar toda su actividad en esta empresa en los años que preludiaban la Guerra civil. Tras su infructuoso intento para recuperar y poner en explotación La Isabela, el conjunto fue anegado por las aguas del embalse de Buendía en 1957.

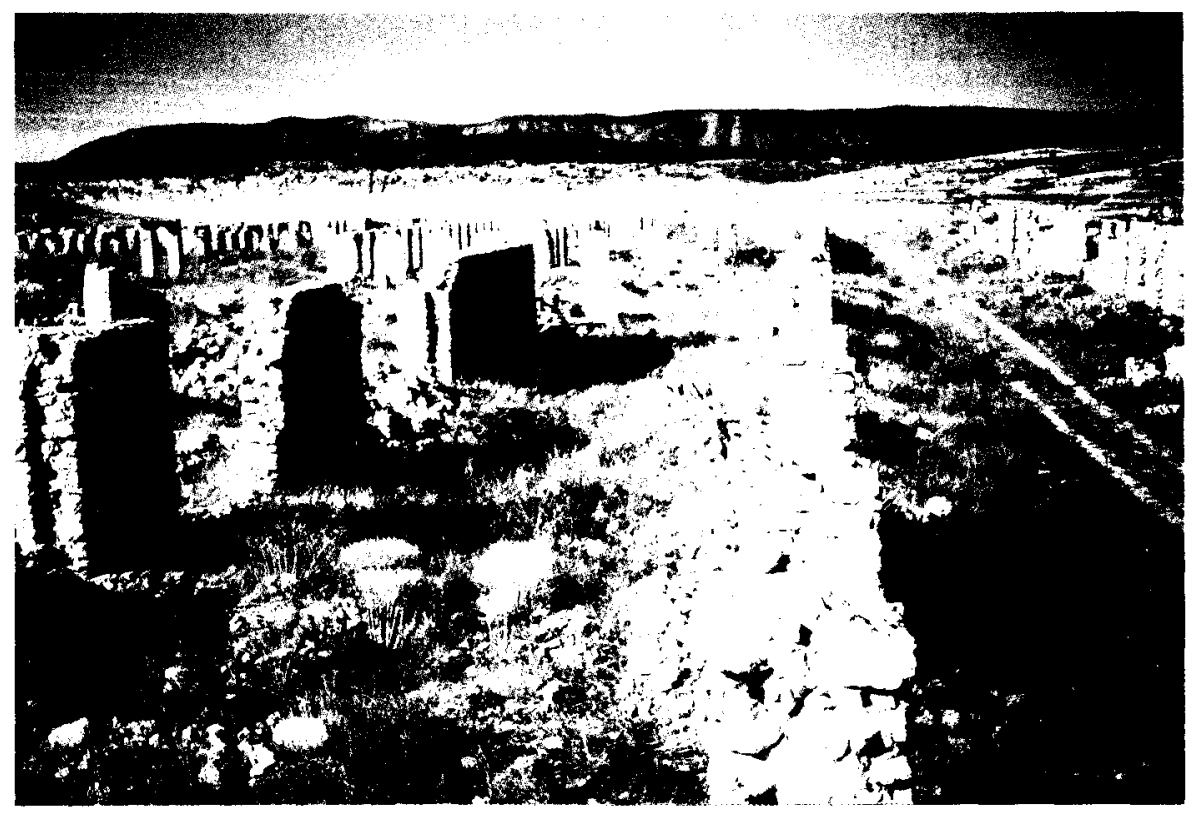

Fig. 3.

Hoy en día los restos de La Isabela pueden ser observados cuando desciende el nivel de las aguas del pantano de Buendía. No obstante, el grado de deterioro de éstos en la actualidad representa una dificultad al

54 Vicente Traver: El Marqués de la Vega Inclán. Castellón, 1965, pág. 216. 
tratar de calibrar la magnitud de un conjunto arquitectónico que, diseñado para obtener rendimiento económico mediante las colonias y el Establecimiento balneario, y para ser lugar de ocio y de reposo de la Monarquía, aunaba el urbanismo cortesano de los Reales Sitios con otras experiencias de poblaciones de nueva planta en España y América, y traducía el modelo europeo de villa termal.

\section{BIBLIOGRAFIÁA}

Aguilera Rojas, J., ibañez Montoya, J., Moreno Rexach, Luis J.: Urbanismo español en América. Centro lberoamericano de Cooperación. Comisaría de Exposición. Madrid, 1979.

Ariza Munoz, Carmen: Los Jardines de Madrid en el siglo Xix. Madrid, 1988.

Artola, Miguel: Los ferrocarriles en España 1844-1943. Madrid, Servicio de Estudios del Banco de España, 1978.

Bernaldo de Quirós, Constancio: Los reyes y la colonización interior de España desde el siglo Xvi al XIx. Madrid, Imp. Helénica, 1929.

Boletín Oficial de Venta de Bienes Nacionales de la provincia de Guadalajara. Años 1871 a 1898.

ButTler, Adrian von (traducción José Luis Gil): Jardines del Clasicismo y el Romanticismo: el jardín paisajista. Soto CABA, Victoria: Jardines de la llustración y el Romanticismo en España. Madrid, Nerea. 1993.

Camara Muñoz, Alicia: “llustración y Sitios Reales en la España del Siglo XVIII". Comunicación en el Congreso El arte en las Cortes europeas del siglo xvili. Comunidad de Madrid, 1987 págs. 177-182.

Caro Baroja, Julio (Director): Arquitectura popular en España: Actas de las jornadas 1-5 de diciembre de 1987. Madrid, Consejo Superior de Investigaciones Científicas, 1990.

Cistellanos de Losada. Basilio Sebastián: La Isabela. Manual del Bañista. Itinerario razonado y descripción del Real Sitio y Baños de La Isabela, mal llamados vulgarmente de Sacedón. Madrid, Sanchiz, 1846.

Cos Gayon, Fernando: Historia Juridica dei Patrimonio Real. Madrid, Enrique de la Riva, 1881

Checa, Fernando, Morán, Miguel: Las casas del Rey. Casas de campo, cazaderos y jardines. Siglos $x$ vi y $x$ vil. Madrid, El Viso, 1986.

Garcia Melero, José Enrique: Influencias artísticas entre España y América. Madrid, MAP. FRE, 1992.

Garcia Melero, José Enrique: Arte español de la llustración y del siglo xix: en torno a la imagen del pasado. Madrid, Ericuentro, 1998

HERNANDO, Javier: Arquitectura en España 1770-1900. Madrid, Manuales de Arte Cátedra, 1989

INFANTE, Fernando: Teatro de la salud, baños de Sacedón hallados del D.D. Fernando Infante, médico de la Reina nuestra Señora, y añadido del doctor D. Juan de la Torre y Valcárcel. Madrid, 1663.

Moleón Gavilanes, Pedro: La arquitectura de Juan de Villanueva: el proceso del proyecto. Madrid, Colegio Oficial de Arquitectos, 1988

Moral Ruiz, J. de: Hacienda y Sociedad en el Trienio Constitucional, 1820-1823. Madrid, Instituto de Estudios Fiscales, 1975.

Moran Martín, M. ${ }^{a}$ Remedios: "Plan de repoblaciones en la segunda mitad del siglo xvIII y primera del XIX». Actas del /l Congreso Histórico sobre nuevas poblaciones de Carlos III en Sierra Morena y Andalucia. Córdoba. Servicio de publicaciones de la Universidad, 1988.

MORENO VILLA, J. : “Planos inéditos de don Isidro Velázquez para una iglesia en La Isabela» Arquitectura, 1932, n. 155, págs. 69-76.

Navascués Palacio, Pedro: "Antonio López Aguado, Arquitecto Mayor de Madrid (1764-1831)" Villa de Madrid. Madrid, n. ${ }^{\circ} 30$, 1970, págs. 84-89.

Oliveras Samitier, Jordi: Nuevas Poblaciones en la España de la llustración. Barcelona, Fundación Caja de Arquitectos, 1998. 
Rueda Hernanz, Germán et al.: La desamortización en la Peninsula Ibérica. Madrid, Marcial Pons, 1993.

SAMBRIcio, Carlos: La arquitectura española de la llustración. Madrid, Coed. C.S. Colegios de Arquitectos de España e Instituto de Estudios de Administración Local, 1986.

SANCHO, José Luis: La Arquitectura de los Sitios Reales. Catálogo Histórico de los Palacios, Jardines y Patronatos Reales del Patrimonio Nacional. Madrid, Patrimonio Nacional, 1995.

TOMAS Y VALIENTE, Francisco: El marco político de la desamortización en España. Barcelona, Ariel, 1989.

Tovar Martin, Virginia: “La casa de campo cortesana en los Sitios Reales". Reales Sitios, n. ${ }^{\circ}$ 67, 1981, págs. 37-44. "Consideración del valor de lo rústico en los Sitios Reales (reinado de Carlos III)». Fragmentos, 12, 13 y 14, 1988, págs. 219-231.

Traver, V.: El Marqués de Vega-Inclán. Primer Comisario de Turismo y Cultura Artística Popular. Castellón, Fundaciones Vega-Inclán, 1965.

VIDAL GALACHE, Florentina: "La beneficencia en Madrid a principios del siglo xIX. El plan de beneficencia de Fernando VIl'. Anales del instituto de Estudios Madrileños, n. ${ }^{\circ} 24,1987$, págs. 133-147. 